\title{
Etude de quelques facteurs de variation du poids à deux mois et des performances de prégavage et de gavage d'oies Landaises en station
}

\author{
R. ROUVIER, A. VRILLON, D. ROUSSELOT-PAILLEY*, P. LARRUE * \\ I.N.R.A., Station d'Amélioration génétique des Animaux \\ Centre de Recherches de Toulouse, B.P. 12, F 31320 Castanet \\ *I.N.R.A., Station expérimentale de l'Oie, Artiguères \\ B.P. $68, F 40002$ Mont-de-Marsan
}

\begin{abstract}
Résumé
La population d'oies Landaises de la Station Expérimentale de l'oie d'Artiguères a été divisée en deux souches (01 et 00) en 1976, dans l'objectif d'une sélection expérimentale pour accroître la production d'oisons et de foie gras. Cet article a pour but d'étudier les paramètres statistiques et zootechniques des deux souches pour les caractères de croissance et de gavage, d'estimer les effets des facteurs de variations dues à l'année, au gaveur, au sexe, à l'interaction gaveur $\times$ sexe.

Les poids corporels à 2 mois et à la mise en pré-gavage, les gains de poids journaliers moyens pendant le pré-gavage et le gavage, les poids de l'oie grasse saignée-plumée et du foie gras, la durée du gavage, sont analysés. Ces caractères ont été mesurés sur 1987 oies des deux sexes, gavées de 1975 à 1979. Le gavage commençait à 20 semaines d'âge, et durait 18 jours en moyenne. Les gains de poids journaliers moyens pendant le pré-gavage et le gavage, les poids de foie gras sont les caractères les plus variables, les poids à 2 mois et à la mise en pré-gavage sont moins variables, la variabilité de la durée du gavage est intermédiaire. L'analyse de variance montre que l'effet année est le plus important. Le poids moyen du foie s'accroît et la durée de gavage se raccourcit, en fonction des années successives. Les poids moyens de foie gras sont de $773 \mathrm{~g}$ et $732 \mathrm{~g}$ dans les deux souches 01 et 00 respectivement en 1979. L'effet gaveur sur le poids du foie est relativement peu important (il rend compte de 2 à 7 p. 100 de la variation totale). Il est cependant très significatif. Les mâles sont plus lourds que les femelles et donnent des poids de foie plus élevés. L'interaction gaveur $\times$ sexe n'est pas significative. Les corrélations phénotypiques obtenues montrent que le gain de poids journalier moyen pendant le gavage pourrait être utilisé pour une prédiction du poids du foie gras, sa corrélation avec la durée du gavage est nulle. Le modèle statistique d'analyse de variance n'explique que 20 p. 100 de la variation totale du poids du foie gras. Il est conclu qu'il sera nécessaire de prendre en compte les effets année, gaveur, sexe dans l'estimation des paramètres génétiques et la sélection pour la production de foie gras.
\end{abstract}

L'amélioration des performances de gavage et foie gras constitue actuellement un des objectifs prioritaires des producteurs d'oies grasses. La sélection génétique est une des voies d'amélioration. A ce point de vue, un plan d'amélioration génétique 
de la population d'oies grises du Sud-Ouest est mis en place à l'échelon national par l'Inter-Profession. La définition d'une méthode de sélection efficace suppose notamment que soient estimés et analysés les effets des sources de variabilité génétique et non génétique des caractères à sélectionner, sur la population animale à améliorer et dans son milieu de production. Les résultats obtenus en Station expérimentale peuvent permettre d'étudier les principaux facteurs de variation, bien qu'ils ne soient pas directement transposables en ferme, et d'expérimenter des méthodes de sélection en vue de mieux connaître le déterminisme génétique des caractères et les progrès génétiques réalisables. MaLLARD (1975) a précédemment analysé les performances de poids à 2 mois et de gavage de 859 animaux de la population d'oies Landaises de la Station d'Artiguères, gavés en 1967, 1969, 1970 et 1971.

On s'était alors limité à tester l'effet combiné année-gaveur et l'effet du sexe ainsi qu'à estimer les paramètres génétiques. Les effets des facteurs non génétiques de variation de ces caractères n'ont pas été quantifiés chez l'oie, à notre connaissance, hormis dans cette étude préliminaire, alors que des valeurs d'estimées de l'héritabilité du poids du foie ont déjà été publiées (STASKo et col., 1976). L'effet gaveur est réputé comme étant important (BLum et col., 1971). On peut penser également à un effet de l'élevage dans lequel sont élevés les oisons jusqu'à leur mise en gavage, à un effet de l'année et de la saison de gavage, en plus d'effets propres à l'animal (date de naissance, âge, sexe).

A partir de 1975 des échantillons d'animaux de la Station expérimentale de l'oie d'Artiguères, de deux souches d'oies Landaises, y sont gavés chaque année en vue de test de la valeur génétique des reproducteurs. Il a semblé utile de présenter ici les résultats, obtenus sur ces animaux de 1975 à 1979, d'une analyse de la variabilité du poids à deux mois et des performances de prégavage et gavage, due aux effets de l'année, du gaveur, du sexe, ainsi que de discuter les corrélations phénotypiques entre les caractères. Dans un article suivant nous étudierons la variabilité génétique de ces caractères.

\section{Matériel animal et caractères mesurés}

La population d'oies Landaises de la Station expérimentale de l'oie d'Artiguères a été constituée en 1958 et 1962 (LARRUE \& MONACHON) à partir de reproducteurs obtenus dans des fermes landaises. Conduite depuis en troupeau fermé, elle a donné lieu aux premières études génétiques de BOYER et col. (1967) et ensuite à différentes analyses de la variabilité (Poujardieu \& Rouvier, non publié). Elle a été séparée à partir de 1976 en deux souches (MONACHON \& Rouvier) codifiées 00 et 01, en vue d'expérimentation en sélection pour améliorer les caractères de production de foie gras et d'oisons. L'objectif est, dans la souche 00 , d'accroître le poids du foie gras obtenu à l'issu du gavage, en maintenant les nombres d'œufs pondus et d'oisons éclos par oie à un niveau optimum; dans la souche 01, d'accroître les nombres d'œufs pondus et d'oisons éclos par oie, en maintenant le poids du foie gras des animaux gavés à un niveau optimum. Les reproducteurs sont conduits en parquets pedigree (25 à 30 dans chaque souche) de 1 jars et 3 oies ou 1 jars et 4 oies, sur caillebotis ou sur sol gravier, jusqu'à la ponte de deuxième année. Cette ponte a lieu en saison naturelle de reproduction, de janvier à juin. Les deux souches sont conduites en générations séparées de deux ans d'intervalle de génération. Leurs reproducteurs ont 
un décalage de un an. En deuxième année de ponte, 4-5 oisons de chaque oie, nés en mars-avril, sont destinés au gavage à partir de la mi-août. Cette opération est réalisée dans la salle de gavage et par les gaveurs de la Station, un échantillon plus limité d'oisons issu de reproducteurs de un an peut dans certains cas être gavé.

\section{A. Technique d'élevage et gavage}

La technique d'élevage des oisons, prégavage et gavage des oies est celle de la Station d'Artiguères (Rousselot, 1974 ; Rousselot et col., 1979).

\section{Conditions du gavage}

Les animaux ont été gavés en 1975 dans deux salles de gavage non climatisées, l'une correspondant à un gavage en épinettes, l'autre en parcs sur litière paille et caillebotis bois. A partir de 1976 les animaux ont été gavés dans une seule salle de gavage climatisée : température entre $16^{\circ}$ et $20^{\circ}$, hygrométrie de 90 p. 100 . Deux déshumidificateurs ont été ajoutés depuis 1978.

La durée maximum du gavage était de 21 jours en 1975, 1976, 1977, 20 jours en 1978 et 19 jours en 1979. Les cinq mêmes gaveurs sont intervenus pendant les cinq années. Un aliment hyperprotidique et supplémenté en choline est distribué pendant une phase de prégavage. Le gavage est fait avec du maïs additionné de 20 p. 100 de féverolles pendant la première semaine (Rousselot et col., 1979). Le maïs distribué est légèrement cuit, salé à raison de 5 à $10 \mathrm{~g}$ de sel et enrichi de graisse après égouttage à raison de 20 à $30 \mathrm{~g}$ de saindoux par $\mathrm{kg}$ de maïs. Le gavage a lieu trois fois par jour. Les animaux qui développent rapidement leur foie gras (ce qui est détecté sur l'animal vivant) sont sacrifiés avant la fin de la durée maximum de gavage définie.

\section{B. Caractères mesurés et analysés}

Les animaux sont pesés individuellement à l'âge de deux mois (P2M), au début du prégavage (PPGAV), au début du gavage. Les poids de l'oie grasse saignée plumée (POG), du foie (PFO) et sa note de qualité, la durée du gavage (DUGAV) sont obtenus. Les gains de poids journaliers individuels moyens pendant le prégavage (GPJPGAV) et pendant le gavage (GPJGAV) ont été calculés, ainsi que le rendement en foie (RENFO) égal au rapport du poids du foie à celui de l'oie grasse, exprimé en pourcentage.

\section{Description de l'échantillon étudié}

Le tableau 1 donne, pour chacune des deux souches, par année, gaveur et sexe, les effectifs d'animaux gavés, les moyennes des dates de naissance, âge des animaux à la mise en prégavage et durées de gavage exprimées en jours. 1447 oisons de la souche 01 et 540 oisons de la souche 00 ont été gavés, soit 1987 oisons au total. Plus de mâles (1 123) que de femelles (864) ont été gavés. Les effectifs sont déséquilibrés par cellule année $\times$ gaveur $\times$ sexe, cependant chacune d'elles est représentée en effectif suffisant pour une analyse statistique. Les animaux destinés au gavage sont choisis aléatoirement au stade oison d'un jour et ensuite à deux mois. La durée de prégavage a été diminuée en 1979 (de 26-27 jours auparavant à 22 jours). 
R. ROUVIER et al.

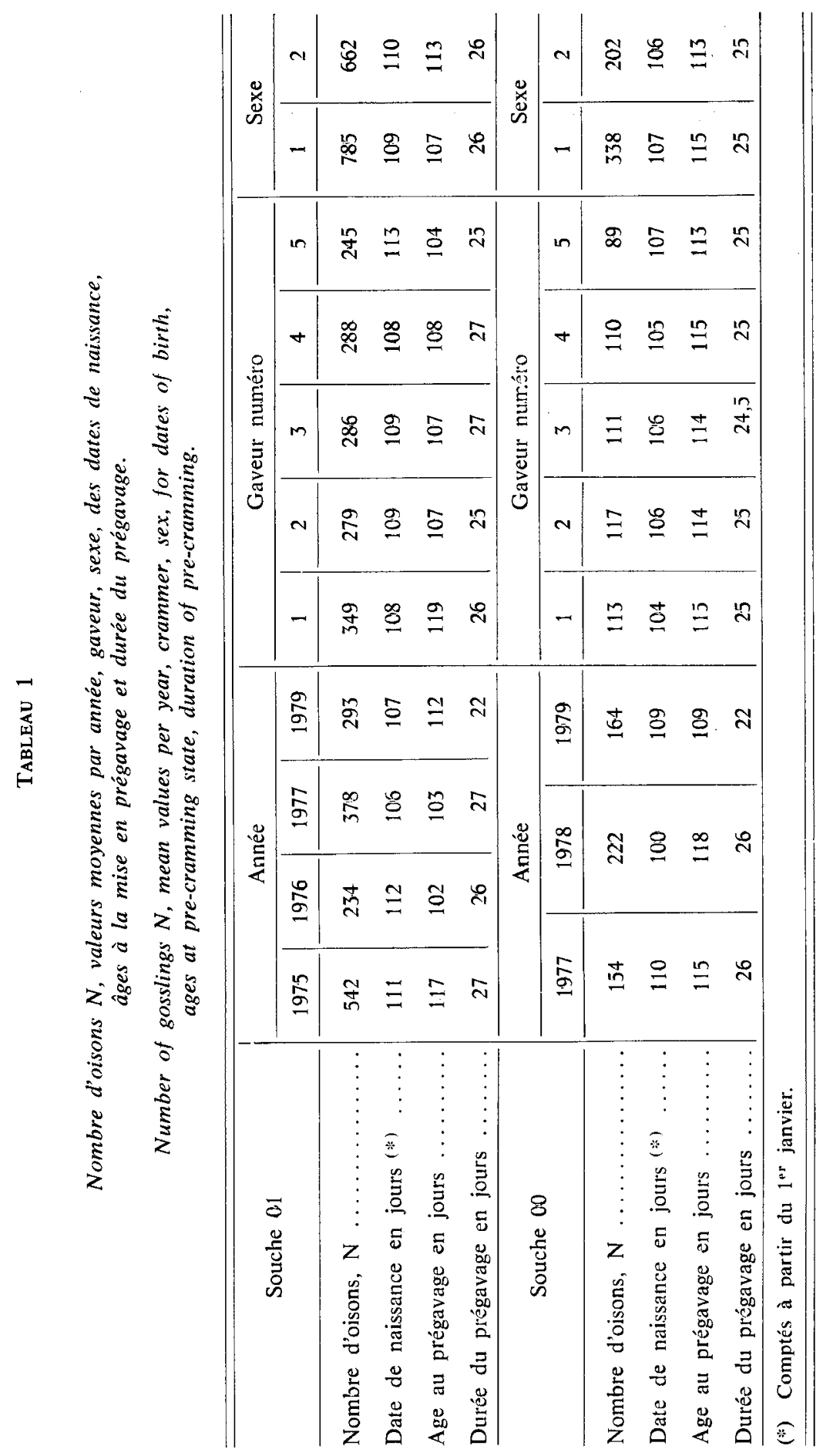




\section{Calculs réalisés et résultats}

La variabilité phénotypique des différents caractères étudiés a été analysée suivant un modèle d'analyse de variance à effets fixés, suivant la méthode des moindres carrés (HaRvey, 1975), avec test et estimation des effets année, gaveur, sexe, et de l'interaction gaveur $\times$ sexe. Les calculs ont été faits au C.T.I. de l'I.N.R.A. Toulouse. Nous n'avons pas analysé la variable qualitative, note de qualité, ni le poids à la mise en gavage qui peut s'obtenir à partir du poids à la mise en prégavage et du gain de poids pendant ce prégavage.

Le tableau 2 donne les moyennes générales, écarts types et coefficients de variation calculés à partir de la variance résiduelle du modèle d'analyse de variance, des différents caractères et de variables relatives au protocole expérimental : date de naissance et âge à la mise en prégavage exprimés en jours à partir du $1^{\text {er }}$ janvier, durée du prégavage. Les animaux sont mis en gavage, en moyenne, à 136 jours dans la souche 01 et 140 jours dans la souche 00 . Cela correspond à des gavages commençant au début de septembre, période encore chaude de l'année dans cette région du Sud-Ouest. Les données n'ont pas été corrigées pour les variations des dates de naissance et âge à la mise en prégavage, dont les coefficients de variation calculée intra année, gaveur, sexe, sont de 8,4 à 13,6 pour 100 .

\section{TABLEAU 2}

Moyennes $(\bar{x})$, écarts-types ( $\sigma)$ résiduels du modèle d'analyse de variance et coefficients de variation (c.v.) en p. 100 des caractères, toutes années regroupées, pour les deux souches 01 et 00.

Means $(\bar{x})$ residual standard deviations ( $\sigma)$ according to the model of variance analysis, coefficient of variation (c.v.) in p. 100 for the characters, all years together, for the two strains 01 and 00 .

\begin{tabular}{|c|c|c|c|c|c|c|c|}
\hline \multirow{2}{*}{ Caractères } & \multicolumn{4}{|c|}{ Souche 01} & \multicolumn{3}{|c|}{ Souche 00} \\
\hline & Unités & $\overline{\mathrm{x}}$ & $\sigma$ & c.v. & $\tilde{\mathbf{x}}$ & $\sigma$ & c.v. \\
\hline P2M & $\mathrm{kg}$ & 4,05 & 0,38 & 9,3 & 4,01 & 0,35 & 8,8 \\
\hline PPGAV & $\mathrm{kg}$ & 4,92 & 0,55 & 11,2 & 5,03 & 0,47 & 9,3 \\
\hline GPJPGAV & $g / j$ & 33,16 & 15,52 & 46,8 & 23,89 & 15,95 & 66,7 \\
\hline DUGAV. & $\mathrm{j}$ & 18,0 & 3,6 & 19,9 & 18,0 & 3,2 & 17,7 \\
\hline GPIGAV & $\mathrm{g} / \mathrm{j}$ & 129 & 33 & 25,7 & 128 & 32 & 25 \\
\hline POG $\ldots$ & $\mathrm{kg}$ & 7,34 & 0,79 & 10,7 & 7,19 & 0,77 & 10,7 \\
\hline PFO .. & $\mathrm{g}$ & 6.10 & 181 & 29,7 & 687 & 188 & 27,4 \\
\hline RENFO & p. 100 & 8,3 & 2,2 & 27,1 & 9,5 & 2,3 & 24,5 \\
\hline Date naissance $\ldots \ldots$. & $\mathbf{j}$ & 109 & 11 & 10,0 & 10.6 & 12 & 11,3 \\
\hline Age au prégavage $\ldots$ & $\mathrm{j}$ & 110 & 15 & 13,6 & 115 & 9,7 & 8,4 \\
\hline Durée du prégavage .. & $\hat{j}$ & 26 & 0,1 & 0.0 & 25 & 0,7 & 0,0 \\
\hline
\end{tabular}

P2M : Poids à deux mois ; PPGAV : Poids à la mise en prégavage ; GPJPGAV : Gain de poids journalier moyen pendant le prégavage; DUGAV : Durée de gavage.

GPJGAV : Gain de poids journal;sr moyen pendant le gavage; POG : Poids de l'oie grasse ; PFO : Poids du foie; RENFO : rendement en foie, en p. 100.

Date de naissance exprimée en nombre de jours depuis le $1^{\mathrm{er}}$ janvier. 


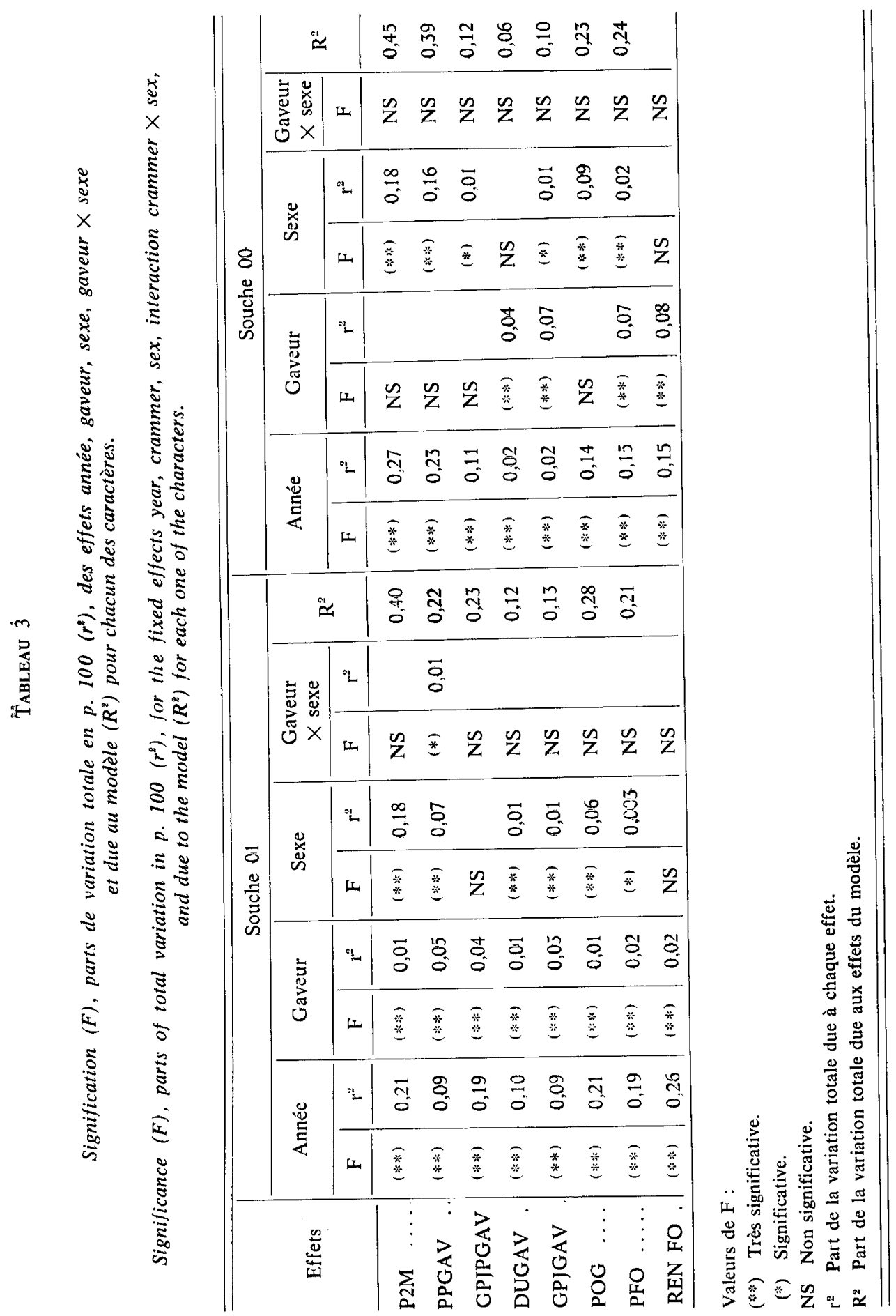


Le tableau 3 donne, pour chacune des deux souches et les huit caractères, la signification des tests $\mathrm{F}$ de Fisher Snedecor de l'analyse de variance pour les. effets fixés année, gaveur, sexe, l'interaction gaveur $\times$ sexe. Nous avons également calculé à partir des sommes de carrés centrés dues à chaque facteur, exprimées en pourcentage de la somme des carrés centrés totale, la part de variation $\left(\mathrm{r}^{2}\right)$ due à chaque effet, lorsqu'il est significatif ou très significatif. La somme de ces valeurs $\mathrm{r}^{2}$, pour un caractère, donne la part de variation $\mathbf{R}^{2}$ expliquée par le modèle. L'effet année est très significatif dans chacune des deux souches et pour l'ensemble des caractères. Il explique la plus grande part de la variation parmi les effets testés. Dans la souche 01 l'effet gaveur est très significatif sur l'ensemble des caractères, et notamment sur les poids à deux mois et à la mise en prégavage. Cela n'est pas le cas dans la souche 00 où l'effet gaveur est très significatif sur la durée du gavage, le gain de poids journalier pendant le gavage, le poids du foie et le rendement en foie, conformément à ce qui est attendu, et n'est pas significatif sur les autres caractères. L'effet sexe est comparable dans les deux souches pour les caractères poids à deux mois et à la mise en prégavage, gain de poids journalier pendant le gavage, poids de l'oie grasse et poids du foie (effets très significatifs ou significatifs), et le rendement en foie (non significatif). Cet effet est différent suivant les deux souches pour le gain de poids journalier pendant le prégavage et la durée du gavage. L'interaction gaveur $\times$ sexe est en général non significative.

Le tableau 4 donne les estimées des effets année, gaveur, sexe, caloulées d'après le modèle d'analyse de variance, exprimées en écarts à la moyenne générale $\hat{u}$ du modèle. La somme des estimées des différentes modalités d'un même facteur n'est pas nulle du fait des conditions de centrage des estimées des effets du programme de calcul qui tient compte du déséquilibre dans les effectifs. L'effet année indique dans la souche 01 , un allègement des animaux à deux mois, à la mise en prégavage, ainsi que de l'oie grasse entre 1975 d'une part (poids plus élevés) et les années suivantes (poids plus faibles). Dans la souche 00 il traduit des poids plus élevés en 1978 par rapport à 1977 et 1979. L'effet année sur la durée du gavage traduit la tendance à la réduction de celle-ci, dans les deux souches, par rapport à la moyenne générale de 18 jours. Il ne traduit pas de tendance nette pour le gain de poids journalier pendant le prégavage. Par contre il y a tendance à une augmentation du poids du foie en 1978 et 1979 par rapport à 1977 dans la souche 00 (par rapport à une moyenne générale de $687 \mathrm{~g}$ ) et en 1979 dans la souche $01:+163 \mathrm{~g}$ par rapport à la moyenne générale de $610 \mathrm{~g}$, soit $773 \mathrm{~g}$. Pour ce même caractère poids du foie, les différences entre les deux mêmes gaveurs extrêmes sont estimées à $99 \mathrm{~g}$ dans la souche 01 , et à $189 \mathrm{~g}$ dans la souche 00 . Les trois gaveurs intermédiaires ne sont pas significativement différents entre eux pour leurs effets sur le poids du foie gras obtenu. Les mâles sont plus lourds que les femelles à deux mois, à la mise en prégavage, ils sont gavés un peu plus longtemps (différence de 0,6 jour) dans la souche 01 , mais pas dans la souche 00 ; ils donnent un foie plus lourd que celui des femelles (différences de $24 \mathrm{~g}$ et $56 \mathrm{~g}$ respectivement dans les souches 01 et 00 ).

Le tableau 5 indique les valeurs des corrélations phénotypiques entre les huit caractères, calculées intra-année, gaveur, sexe, pour chacune des deux souches. Toute corrélation supérieure en valeur absolue à 0,05 est significative dans la souche 01 , et supérieure à 0,08 est significative dans la souche 00 . 


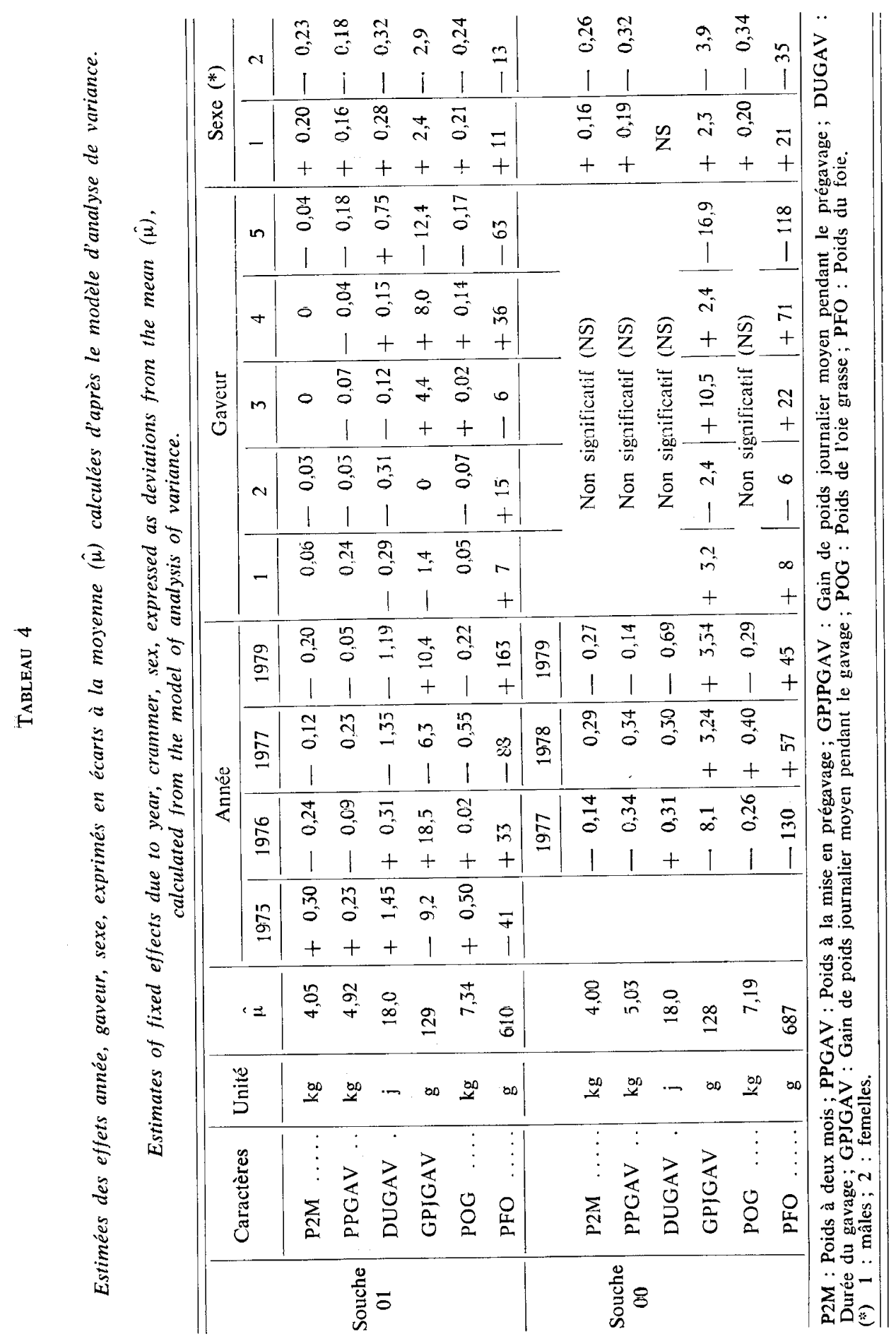




\section{TABLEAU 5}

Corrélations phénotypiques calculées intra année, gaveur, sexe.

Phenotypic correlations calculated within year, crammer, sex.

\begin{tabular}{|c|c|c|c|c|c|c|c|c|}
\hline \multicolumn{9}{|l|}{ Souche 01} \\
\hline Caractères & 1 & 2 & 3 & 4 & 5 & 6 & 7 & 8 \\
\hline $\begin{array}{ll}1 & \text { P2M ...... } \\
2 & \text { PPGAV } \\
3 & \text { GPJPGAV } \\
4 & \text { DUGAV } \\
5 & \text { GPJGAV } \\
6 & \text { POG } . . . \\
7 & \text { PFO } \ldots . \\
8 & \text { RENFO }\end{array}$ & 1,00 & $\begin{array}{c}0,63 \\
1,0\end{array}$ & $\begin{array}{r}0,18 \\
-0,23 \\
1,00\end{array}$ & $\begin{array}{c}0 \\
-0,04 \\
0,11 \\
1,00\end{array}$ & $\begin{array}{r}0,29 \\
0,26 \\
-0,10 \\
-0,25 \\
1,00\end{array}$ & $\begin{array}{l}0,62 \\
0,58 \\
0,35 \\
0,34 \\
0,54 \\
1,00\end{array}$ & $\begin{array}{l}0,20 \\
0,11 \\
0,17 \\
0,08 \\
0,50 \\
0,51 \\
1,00\end{array}$ & $\begin{array}{r}0 \\
-0,13 \\
0,03 \\
-0,06 \\
0,33 \\
0,13 \\
0,91 \\
1,00\end{array}$ \\
\hline \multicolumn{9}{|l|}{ Souche 00} \\
\hline Caractères & 1 & 2 & 3 & 4 & 5 & 6 & 7 & 8 \\
\hline $\begin{array}{ll}1 & \text { P2M ...... } \\
2 & \text { PPGAV } \\
3 & \text { GPIPGAV } \\
4 & \text { DUGAV } \\
5 & \text { GPJGAV } \\
6 & \text { POG .... } \\
7 & \text { PFO ..... } \\
8 & \text { RENFO }\end{array}$ & 1,00 & $\begin{array}{l}0,54 \\
1,00\end{array}$ & $\begin{array}{r}0,16 \\
-0,30 \\
1,00\end{array}$ & $\begin{array}{c}0 \\
0,06 \\
-0,05 \\
1,00\end{array}$ & $\begin{array}{r}0,19 \\
0,11 \\
0,01 \\
-0,30 \\
1,00\end{array}$ & $\begin{array}{l}0,55 \\
0,57 \\
0,27 \\
0,35 \\
0,48 \\
1,00\end{array}$ & $\begin{array}{r}0,18 \\
0,19 \\
0,07 \\
-0,03 \\
0,42 \\
0,42 \\
1,00\end{array}$ & $\begin{array}{c}0 \\
0 \\
-0,05 \\
-0,20 \\
0,26 \\
0,05 \\
0,92 \\
1,00\end{array}$ \\
\hline
\end{tabular}

Corrélations significatives : supérieures en valeurs absolues à $r=0,05$ (souche 01 ) et $r=0,08$ (souche 00).

P2M : Poids à deux mois ; PPGAV : Poids à la mise en prégavage; GPJPGAV : Gain de poids journalier moyen pendant le prégavage ; DUGAV : Durée dû gavage; GPJGAV : Gain de poids journalier moyen pendant le gavage; POG : Poids de l'oie grasse; PFO : Poids du foie; RENFO : rendement en foie.

\section{Discussion}

L'effet de l'âge des animaux n'a pas été pris en compte dans le modèle, ni celui de la série de gavage intra-année. Les âges moyens à la mise en prégavage varient peu (tabl. 1) entre années, gaveurs et sexes. Leurs corrélations avec l'ensemble des caractères étudiés, calculées intra-année, gaveur et sexe ne sont pas significatives, sauf des corrélations positives (souche 01) entre l'âge à la mise en prégavage d'une part, et d'autre part le poids à la mise en prégavage $(r=0,38)$, le poids de l'oie grasse $(r=0,22)$. Ces variations d'âge à la mise en prégavage n'influent pas sur le poids du foie. Chaque année, les animaux des deux souches ont été gavés en deux 
séries successives, les mêmes cinq gaveurs intervenant dans toutes les séries. L'effet série n'a pas été considéré dans le modèle, l'effet de sa variation est donc inclus dans les variances résiduelles.

\section{A. Valeurs moyennes et variabilité phénotypique}

Ces valeurs sont indiquées dans le tableau 2. L'objectif n'est pas de comparer les deux souches dont les orientations de sélection différentes sur le poids du foie ont été définies récemment. Elles ont les mêmes valeurs moyennes pour le poids à deux mois $(4,05$ et $4,01 \mathrm{~kg}$, respectivement), le poids au prégavage $(4,92$ et $5,03 \mathrm{~kg})$, la durée du gavage (18 jours), le gain de poids journalier moyen pendant le gavage (129 et $128 \mathrm{~g} / \mathrm{j})$, des valeurs peu différentes pour le poids de l'oie grasse saignée plumée $(7,34$ et $7,19 \mathrm{~kg})$. Si elles semblent différer pour le poids moyen du foie gras $(610 \mathrm{~g}$ et $687 \mathrm{~g}$ ) et corrélativement le rendement en foie, cela est lié à l'effet défavorable de l'année 1975 où seule la souche 01 a été gavée. Cette différence des moyennes générales des poids du foie provient de l'amélioration de la technicité des gaveurs et des conditions de salle de gavage à partir de 1978 et 1979 , années pour lesquelles les poids de foie obtenus sont de $744 \mathrm{~g}$ (souche 00), $773 \mathrm{~g}$ (souche 01) et $732 \mathrm{~g}$ (souche 00).

Les coefficients de variation des poids à deux mois, à la mise en prégavage, de l'oie grasse, sont ceux que l'on peut attendre pour des caractères de croissance $(9$ à 11 p. 100). Par contre, le coefficient de variation du gain de poids journalier pendant le prégavage est élevé ( 46,8 et 66,7 p. 100), indiquant que les oies répondent de façon très variable à l'alimentation prégavage.

Le poids du foie est très variable (c.v. : 29,7 et 27,4 p. 100), ainsi que le gain de poids journalier moyen pendant le gavage (c.v. 25,7 et 25 p. 100), et plus que la durée de gavage (c.v. : 19,9 et 17,7 p. 100). La plupart des oies sont gavées en 14 à 22 jours. Cela indique une aptitude à un développement rapide du foie gras, le gavage de certaines oies étant arrêté très tôt pour éviter des foies trop gros (absence de corrélation entre poids du foie et durée de gavage, tabl. 5). La variabilité du rendement en foie est liée à celle du poids en foie.

En ce qui concerne les performances d'oies d'autres races, BIELINSKI et BIELINSKA (1969) comparent des performances de gavage d'oies de races Poméranienne, Zatorsk, Blanche d'Italie, Gorkov. Ils concluent que les oies Blanche d'Italie présentent les meilleurs aptitudes pour la production de foie gras. Pour des oies grasses (saignées, plumées) pesant en moyenne $8,8 \mathrm{~kg}$ (écart type 1,20 ) ils obtiennent une moyenne de poids de foie gras de $652 \mathrm{~g}$. L'écart type de $212 \mathrm{~g}$ indique un coefficient de variation de 32,5 p. 100 , comparable donc à celui de nos résultats. Par contre, le rendement foie gras (poids du foie/poids de l'oie grasse) n'est que de 7,03 p. 100 (8,3 et 9,5 p. 100 dans nos résultats).

Ces résultats sont confirmés par BoBrowska (1975) qui compare 4 types régionaux d'oies de Poméranie, 5 types régionaux d'oies blanches améliorées et des oies Blanches Italiennes. BIELINSKI (1973) indique que les oies Blanches Italiennes sont préférables aux Poméraniennes pour la production du foie gras.

BOYER (1969) avait obtenu dans la population d'oies Landaises étliciée, contrôlée en 1967, une moyenne de poids de foie de $684 \mathrm{~g}$ (rendement foie de $8,6 \mathrm{p}$. 100). 
Dans une anlyse préliminaire précédente (MALlaRD, 1975), les poids moyens de foie obtenus étaient, pour les deux sexes confondus, de $657 \mathrm{~g}$ (1967), $664 \mathrm{~g}$ (1969), $554 \mathrm{~g}(1970)$ et $603 \mathrm{~g}$ (1971). Les résultats indiquent donc une amélioration du poids du foie dans cette souche à partir de 1978. D'après les résultats publiés, seule l'oie Blanche Italienne, étudiée par les chercheurs Polonais, semble avoir donné des résultats comparables pour le poids du foie, mais avec une durée de gavage un peu plus longue, et un poids de l'oie grasse plus élevé. La moyenne de poids de fo:e que nous obtenons en Station est du niveau de celle observée en ferme avec loie grise du Sud-Ouest de la France, avec les meilleurs gaveurs (Rouvier et $M^{\text {me }}$ SAZY E., non publié), et des oies également plus lourdes ainsi qu'une durée de gavage plus longue. La durée de gavage pourrait donc être réduite en pratique, ce qui présenterait un intérêt économique pour cette production. Par contre, les résultats indiquent l'existence d'une variabilité individuelle de la réponse à l'alimentation pour la formation de foie gras. Celle-ci serait à analyser et à exploiter par l'alimentation et la sélection .L'on sait que BLUM \& LECLERQ (1970) ont montré l'intérêt, dans le but de favoriser la synthèse de la trame protéique du foie et de favoriser une hypertrophie de cellules à membranes résistantes (qualité des foies) d'un aliment de prégavage et de début de gavage ( 8 jours) riche en protéines ou en choline ou les deux à la fois (Rousselot-Pailley, 1974). Il semble que l'utilisation de ces techniques d'alimentation et l'accroissement de la technicité de gavage, aient permis l'amélioration notable des performances foie gras de cette population d'Artiguères qui se manifeste en 1978 et 1979.

\section{B. Facteurs de variation}

Les facteurs de variations des performances de croissance et de gavage, dues simultanément à l'année, au gaveur et au sexe, ont été très peu étudiés jusqu'à présent chez l'oie. Babile et col. (1980) étudient la production de foie gras de canards de Barbarie mâles. Un modèle d'analyse de variance prenant en compte trois effets principaux (4 types génétiques, 2 gaveurs, 4 classes de qualité commerciale), trois interactions entre ces trois facteurs 2 à 2, et la régression du caractère mesurée sur la durée de gavage, permet de rendre compte de 36 p. 100 de la variance du poids de foie. La valeur du F de l'effet gaveur, très significative, est supérieure à celle de l'effet type génétique, mais inférieure à celle de la classe de qualité également très significative. Nous n'avons pas ici introduit la note de qualité comme facteur du modèle, considérant d'une part que la qualité mesurée par la fonte lipidique des foies à la cuisson est un caractère lié à l'animal, et que d'autre part elle est actuellement mal exprimée par la note de classification commerciale. La durée de gavage est considérée comme une variable, bien qu'elle soit limitée supérieurement par le protocole expérimental.

Le modèle d'analyse de variance utilisé rend compte de 40 et 45 p. 100 de la variation de poids à deux mois, pour les deux souches respectivement, ce qui est dû aux effets de l'année et du sexe. Il rend compte d'une part plus faible de la variation des autres caractères, notamment des poids de foie $\left(R^{2}: 0,21\right.$ et 0,24$)$.

L'effet année est le plus important (valeurs de $\mathrm{r}^{2}$, tabl. 3). Le gain de poids journalier moyen pendant le gavage est le caractère le moins affecté. L'effet année sur la durée du gavage est lié à la décision de réduction progressive de la valeur maximum de celle-ci. Les deux faits qui apparaissent plus clairement sont la tendance à l'allègement des animaux gavés dans la souche 01 d'une part, l'accroissement du 
poids du foie à partir de 1978 d'autre part. La mise en évidence de cet effet année montre que, même à protocole expérimental constant, il faut en tenir compte dans l'interprétation des résultats. Celui-ci tient compte de l'évolution génétique des populations, de l'influence des facteurs climatiques et alimentaires qui peuvent intervenir sur la croissance et la production de foie gras.

L'effet gaveur sur le poids à 2 mois et le poids à la mise en prégavage permet de tester le protocole expérimental quant à la répartition des animaux entre les différents gaveurs en fonction de leur croissance pondérale. Bien que celle-ci se fasse aléatoirement, il est significatif (mais faible en valeur relative) dans le cas de la souche 01. Il ne l'est pas dans la souche 00. Pour une analyse de cet effet gaveur, il conviendrait donc d'affecter aux gaveurs des échantillons d'animaux de même poids moyen à deux mois, ou d'utiliser ce caractère comme covariable dans le modèle statistique d'analyse de variance. L'effet gaveur sur la durée du gavage est faible, significatif dans la souche 01 , mais non significatif dans la souche 00 . Pour ce caractère phénotypiquement variable, les gaveurs gavent en moyenne pendant la même durée. Cela est lié, ici, à la fixation d'une durée maximum de gavage qui est courte. Les effets gaveurs sur le gain de poids journalier moyen pendant le gavage, le poids de l'oie grasse, le poids du foie sont relativement faibles, quoique très significatifs, sauf pour le poids de l'oie grasse dans la souche 00 . Pour le poids du foie ils ne représentent que 2 et 7 p. 100 de la variation totale de ce caractère. Cet effet gaveur, pour des animaux d'une même souche, comparables par ailleurs, est donc moins important qu'on ne le pense, dans le cas où tous les gaveurs sont expérimentés. Ces résultats tiennent à l'homogénéité des techniques d'élevage et gavage à la station. Cependant, compte tenu de la forte variabilité phénotypique des poids de foie, les différences entre effets moyens des gaveurs peuvent être importantes en valeurs absolues (tabl. 4). Cet effet gaveur doit donc être pris en compte dans tout protocole expérimental ou d'études génétiques.

L'effet sexe est très significatif, dans les deux souches, sur les caractères de croissance pondérale jusqu'au prégavage, significatif ou très significatif suivant la souche sur la vitesse de croissance journalière moyenne pendant le gavage et sur le poids du foie. La différence de poids à deux mois éntre mâles et femelles, de 400 ou $420 \mathrm{~g}$ en faveur des mâles, représente $10 \mathrm{p} .100 \mathrm{du}$ poids corporel. Moins de femelles que de mâles sont gavées, plus de femelles que de mâles étant nécessaires pour le renouvellement des troupeaux, mais l'échantillonnage réalisé à la mise en prégavage ne biaise pas cette comparaison de móyennes. Cette différence entre sexes se maintient ou s'accroît légèrement dans la souche 00 pour le poids à la mise en prégavage. Le gain de poids journalier pendant le gavage est supérieur chez les mâles qui ont un foie plus lourd. Cependant, la différence entre sexes pour le poids de l'oie grasse (450-540 g pour les deux souches respectivement) est en grande partie réalisée dès l'âge de deux mois. Les femelles donnent des rendements en foie gras comparables à ceux des mâles et des poids de foie gras un peu inférieurs (la différence représente 4 à $8 \mathrm{p} .100$ du poids moyen du foie dans les deux souches 01 et 00 respectivement).

L'effet du sexe sur la croissance pondérale de l'oie a été mis en évidence par plusieurs auteurs sur différentes races, mais de façon plus qualitative que quantitative. Lès résultats concernant son effet sur le poids du foie sont plus rares et ne sont pas toujours significatifs : Bielinski (1967), Gком et col. (1967), Bögre (1969), BiELiNSKi K. \& BiELINSKA K. (1969), BielinsKA K. et col. (1975). BACZKowsKa (1967, 
1969) indique que la différence de croissance entre mâles et femelles se manifeste dès l'âge de quatre semaines.

En conclusion, le dimorphisme sexuel en poids vif en faveur des mâles se manifeste pleinement à l'âge de 8 semaines, à la fin de la période de croissance rapide (Rousselot-Pailley, 1974). La différence entre sexes s'accroît lentement par la suite. Nous mettons cependant nettement en évidence une croissance plus rapide des mâles pendant le gavage, pour ces oies gavées à l'âge de 20 semaines, qui donnent des foies un peu plus lourds que ceux des femelles en moyenne, sans différence de rendement en foie.

\section{Corrélations}

L'étude des corrélations phénotypiques calculées intra-année, gaveur, sexe, pour éliminer les effets de ces facteurs de variation (tabl. 5) doit permettre notamment de rechercher des caractères prédicteurs du poids du foie. Les corrélations calculées pour chacune des deux souches peuvent être considérées comme homogènes. Intra-souche, le poids du foie dépend linéairement du gain de poids journalier pendant le gavage $(\mathrm{r}=0,50$ et 0,42 ) ainsi que du gain de poids total pendant le gavage (corrélations du même ordre de grandeur). Ce poids du foie est linéairement indépendant de la durée de gavage. Il présente des corrélations faibles (inférieures ou égales à 0,20 ) avec les poids à deux mois, à la mise en prégavage, et le gain de poids journalier pendant le prégavage. Par contre, le poids de l'oie grasse présente des corrélations plus élevées à la fois avec le poids du foie $(r=0,4$ à 0,5$)$ et avec ces caractères pondéraux. La corrélation entre le poids de l'oie grasse et le poids à deux mois, notamment, a des valeurs $\mathrm{r}=0,55$ et $\mathrm{r}=0,62$. Ce sont donc les animaux à forte capacité d'accroissement de poids vif pendant le gavage qui donnent les foies les plus lourds. Cette aptitude n'est pas prévisible avec suffisamment de précision à partir des caractères de croissance plus précoces étudiés. Par contre, des poids à deux mois plus élevés s'accompagnent de poids plus élevés de l'oie grasse, et probablement de paletot.

Contrairement à nous, STASKo \& MAJNA (1976) étudiant quatre types génétiques d'oies gravées depuis l'âge de 162 jours, pendant 33 jours après une période préparatoire de 34 jours, trouvent des corrélations phénotypiques plus élevées entre poids corporel au début de la période préparatoire et poids du foie. Ils trouvent également des corrélations fortes entre poids corporel au début du gavage et poids du foie. Les poids de foie obtenus ne sont pas indiqués. Par contre, Grom et col. (1967) n'avaient pas trouvé ces corrélations. BoBrowsKa (1975), dans le travail déjà cité, trouve que le poids du foie est très significativement corrélé avec le poids corporel au début de l'engraissement $(\mathrm{r}=0,50)$, la durée d'engraissement $(r=0,44)$ et la quantité de nourriture ingérée $(r=0,42)$. Les deux sexes ne sont pas distingués et la variabilité des poids est grande, ce qui peut entraîner une covariation plus grande. BIELINSKI (1967) trouve également, chez l'oie Blanche Italienne, un coefficient de corrélation entre poids corporel et poids de foie gras, $r=0,52$. Ce résultat est confirmé par BIELINSKi (1973) qui indique une corrélation entre poids corporel avant gavage et poids du foie, ainsi qu'avec le poids du tissu maigre du foie $(r=0,86)$. Par contre Stasko \& Majna (1968) ne trouvent pas de relation génétique entre le poids du foie et le gain de poids corporel pendant la première période d'engraissement. Nos résultats devront donc être confirmés pour savoir s'ils sont caractéristiques de cette population d'oie Landaise et de ses conditions 
d'élevage, prégavage et gavage. Les relations entre croissance corporelle et formation du foie gras devront être approfondies. Il serait plus intéressant d'étudier les corrélations entre quantité de maïs ingéré et durée de gavage, production de foie gras, de graisse de dépôt et de viande, pendant le gavage. En effet, une capacité d'accroissement de poids vif plus élevée pendant le gavage pourrait être liée à une capacité de plus forte ingestion de maïs dont il faudrait étudier la cinétique et l'utilisation pour produire le foie gras et les différents tissus. Les travaux précurseurs de BIELiNSKA \& BiELINSKI (1971) qui étudient les relations entre quantité de maïs ingéré pendant le gavage, durée du gavage et poids du foie gras obtenu, vont à l'appui de cette hypothèse. Ils trouvent sur 115 jars de race Blanche d'Italie gavés, âgés de 6 à 7 mois, une corrélation $\mathrm{r}=0,797$ entre la quantité de maïs consommé pendant le gavage et le gain de poids, et $r=0,693$ avec le poids du foie. Pour la corrélation entre le gain de poids et le poids du foie, nos résultats confirment ceux de ces auteurs qui trouvent $r=0,583$. S'ils ne trouvent pas de corrélation significative dans ce cas entre le poids corporel au début du gavage et le poids du foie $(r=0,068)$, par contre la quantité de maïs ingéré est fonction de ce poids en début de période $(r=0,549)$. Mis à part l'âge plus élevé des animaux, les résultats de ces auteurs sont comparables aux nôtres, notamment pour la durée de gavage ( 24,3 jours en moyenne) et le poids du foie gras obtenu (778 $\mathrm{g}$ en moyenne), le poids moyen des animaux à la mise en gavage étant de $5,38 \mathrm{~kg}$. Ces auteurs concluent que le résultat du gavage et en particulier le poids du foie gras obtenu dépendent de l'aptitude des oies à consommer de grandes quantités de maïs.

\section{Conclusion}

Nous avons utilisé les résultats de gavage servant au test habituel des reproducteurs de deux souches d'oies Landaises de la Station expérimentale de l'Oie d'Artiguères pour en déterminer les paramètres statistiques et zootechniques, dans la période récente de 1975 à 1979, ainsi que pour étudier l'importance des facteurs de variations dus à l'année, au gaveur, au sexe et à l'interaction gaveur $\times$ sexe. Il s'agit d'une étape préliminaire pour des expérimentations en sélection sur la production de foie gras et d'oisons. Cette étude, qui porte sur un effectif d'animaux important, apporte des résultats originaux comme l'indique la revue bibliographique qui a été faite. L'année est le facteur majeur de variation. L'effet année sur le poids du foie traduit une augmentation du poids moyen des foies des animaux à partir de 1978. Celle-ci semble être due à l'amélioration des techniques d'alimentation (prégavage et gavage) et de la technicité des gavages, plutôt qu'à la sélection sur le poids du foie, notamment pour la souche 01 . L'effet année est également important sur le poids à deux mois, ce qui entraîne des effets corrélés sur les caractères pondéraux (poids à la mise en prégavage, poids de l'oie grasse). Il semble traduire dans la souche 01 une évolution allant dans le sens d'une diminution du poids à deux mois et du poids de l'oie grasse. Il restera à confirmer, dans les générations ultérieures, si cette souche a tendance à s'alléger comme conséquence de la sélection sur le nombre d'œufs pondus et mis en incubation. L'effet année sur le gain de poids journalier pendant le gavage peut traduire des variations dans la qualité de l'aliment de gavage. En conclusion, cet effet année peut avoir de multiples raisons, y compris climatiques. Il doit donc être pris en compte, en tant qu'effet global, dans toute étude expérimentale. Jusqu'à présent l'effet gaveur n'avait pas été mis en évidence quantitativement. Il est ici 
relativement faible dans la variation qu'il entraîne, par rapport à la variation totale. Compte tenu de la grande variabilité du poids du foie, il peut cependant être important en valeur absolue sur les valeurs moyennes. Il est évidemment lié à l'échantillon de gaveurs considéré. Même en station, il sera nécessaire, dans l'estimation de la valeur génétique des reproducteurs, de corriger les données pour les effets gaveurs sur les moyennes. Il sera également nécessaire de les corriger pour un effet sexe, les mâles donnant un foie gras significativement plus lourd que celui des femelles, ce que les études antérieures n'avaient pas mis en évidence. Le dimorphisme sexuel en faveur des mâles pour les caractères de poids corporel, très significatif dans ces souches, correspond à ce qui a été trouvé sur d'autres races d'oies, sauf pour le gain de poids pendant le gavage qui n'avait pas été étudié jusqu'à présent.

Sur d'autres races, des corrélations entre poids corporel et poids du foie gras ont été trouvées. Celles-ci sont faibles dans ces deux souches, sauf avec le poids de l'oie grasse. Par contre le gain de poids journalier moyen pendant le gavage est corrélé avec le poids du foie $(r=0,5)$. La durée de gavage peut être réduite chez l'oie, tout en permettant l'obtention d'un poids de foie de 700-800 g. L'ensemble de ces relations devra être vérifié sur le plan génétique.

La note de qualité du foie n'a pas été étudiée. La qualité est un caractère essentiel à considérer qui doit être défini plus objectivement, notamment dans sa relation avec le poids du foie, pour pouvoir définir un objectif de sélection sur le critère poidsqualité du foie gras.

La sélection, qu'elle soit expérimentale en station ou pratique en ferme, devra se faire intra-année, prendre en compte les effets élevages-gaveurs, sexe, et se baser sur des objectifs et critères définis. La présente analyse de la variabilité, quoique limitée à quelques caractères zootechniques, et ses résultats devraient contribuer à cette définition.

Reçu pour publication en décembre 1981.

\section{Remerciements}

Nous remercions B. Poujardieu pour son aide dans l'utilisation des modèles statistiques et la discussion des résultats ainsi que J.C. Blum pour ses lectures critiques qui ont permis d'améliorer le manuscrit.

\section{Summary}

Study of some factors of variation of the weight at two months and of the preforce feeding and force feeding of Landaise goose in station

A population of Landaise goose from the Experimental Research Station d'Artiguères was divided into two strains $(01$ and 00$)$ in a selection experiment to increase both gosling and fat liver production. The purpose of this paper is to study some statistical and production parameters of these two strains for growth and force-feeding traits and to estimate the variability due to year, sex and crammer effects as well as crammer $\times$ sex interaction effect. Individual body weight at 2 months and at the beginning of force-feeding, fattened goose weight, fat liver weight, the duration of force-feeding and the average daily weight gains during both the pre-force-feeding and the feeding periods are analysed. The study relates 
to records of 1987 geese of both sexes during the years 1975 to 1979 . Force feedingcommenced at 20 weeks of age and was continued for an average of 18 days. Daily weight gains in the pre-force-feeding as well as during the force-feeding periods and fat liver weights are the most variable traits. Two-months body weight and weight at the start of force-feeding are less variable while the duration of force-feeding is intermediary. The analysis of variance shows that year effect is the most important. Mean fat liver weight increased while the duration of force-feeding period decreased in successive years. Mean fat liver weights in 1979 were $773 \mathrm{~g}$ and $732 \mathrm{~g}$ for strains 01 and 00 respectively. Crammer effect on fat liver weight is relatively unimportant (accounting for only 2 to 7 p. 100 of the total variation) but is highly significant. Males are heavier than females and yield heavier fat livers. The crammer $\times$ sex interaction is not significant. Phenotypic correlations show that average daily weight gains during force-feeding may be used to predict fat liver weights but there was no correlation with the duration of force feeding. The statistical model of the analysis of variance explains only 20 p. 100 of the total variation of fat liver weight. It is concluded that the fixed effects of year, crammer and sex must be taken into account in order to estimate genetic parameters and to select for fat liver production.

\section{Références bibliographiques}

Babile R., Matheron G., Delpech P., Farran M.T., 1980. La production de foie gras de canard de Barbarie mâle : analyse de différents facteurs de variation. Ann. Zootech., 29, 265-276.

Baczkowska H., 1967. Etude de la croissance des oisons. Journées de l'oie, Jouy-en-Josas, France. Document à diffusion restreinte.

Baczkowska H., 1969. Etude de la croissance des oisons. L'alimentation et la vie, 57, 131.

BIELINSKI K., 1967. Relationship between the weight of geese before fattening and the amount of foie gras. Roczn. Nauk. roln., Ser. B, 89, 413-418.

BieliNSKi K., BielinsKa K., 1969. Analyse des races d'oies autochtones et étrangères élevées en Pologne et les résultats de gavage de ces races à foie gras. L'alimentation et la vie, 57, 111-113.

BielinsKa K., Bielinski K., 1971. Badania nad intensywnoscia tuczu gesi nastluszczone watroby. Roczniki Nauk Rolniczych, B 93-4, 55-64.

BIELINSKI K., 1973. Problems of the production of foie gras in geese. Naus Chov, 33, 258-261.

Bielinska K., Pujsko K., Bielinski K., KaszYnski J., 1975. Effect of age on yield, chemical composition and nutritional value of breast muscles in broiler geese. Postepy Drobiarstwa, 17, 5-15.

Blum J.C., Leclerce B., 1970. Facteur favorable à la formation du foie gras chez l'oie : la distribution d'un régime enrichi en choline pendant la période précédant le gavage. Ann. Zootech., 19, 347-351.

Blum J.C., Monachon G., Leclere B., 1971. Liver steatosis of force-fed geese as influenced by the protein level of the diet. Acta .Vet. Acad. Sci. Hung., 21, 307-311.

BobrowsKa W., 1975. The suitability of regional type of geese for foie gras production. Roczniki Nauk Rolniczych, B 97, 2, 39-52.

Bogre J., 1957. Heritability $\left(\mathrm{h}^{2}\right)$ of liver size in fattened geese. Agrartud. egy. Allattenyeszt Karának Közl (Gödöllö-Budapest), 3, 13-25.

BOGRE J., 1969. Investigation of the growth stages of the regional races of the Hungarian goose and its hybrids. In Phaeno-analysis and quantitative inheritance. Ed. by Gy. Fabian. Budapest : Akademiai Kiado, 159-170.

Boyer J.P., Larrue P., Monachon G., 1967. Antagonisme génétique entre l'aptitude à l'engraissement du foie et la fécondité chez l'oie. Journées de l'oie, Jouy-en-Josas, France. Document à diffusion restreinte.

BOYER J.P., 1969. Antagonisme génétique entre production de foie gras et production d'oisons. L'alimentation et la vie, 57, 113-114. 
Grom A., Stasko J., Masar T., 1967. Quelques paramètres génotypiques et phénotypiques des oies élevées en Tchécoslovaquie. Journées de l'oie „Jouy-en-Josas, France. Document à diffusion restreinte.

Grom A., Stasko J., Masar T., 1969. Quelques paramètres génotypiques et phénotypiques des oies élevées en Tchécoslovaquie. L'alimentation et la vie, 57, 115-116.

Harvey W.R., 1975. Least squares analysis of data. Agricultural Research Service, U.S. Department of Agriculture, 1st printing 1960.

Mallard Y., 1975. Etude des performances de gavage chez l'oie. Mémoire de fin d'études, E.N.S.A., Toulouse.

Rocsselot-Pailley D., 1974. L'élevage de l'oie. Son évolution grâce à la Recherche. Bulletin Technique d'Information, 292, 1-23.

Rousselot-Pailley D., Larrue P., Monachon G., 1979. Les derniers progrès dans l'élevage de l'oie à Artiguères. Station expérimentale de l'Oie, Artiguères. Document à diffusion restreinte.

Rouvier R., Vrillon A., Rousselot-Pailey D., Larrue P., 1982. Note sur l'efficacité thécrique d'un indice de sélection sur collatéraux pour le foie gras de l'oie Landaise. Ann. Génét. Sél. anim., 14, 245-252.

Stasko J., Masar T., 1968. Studies on the heritability and relationship of some growth and fattening capacity indexes in geese. Zivoc. Vyroba, 13, 455-463.

Stasko J., Grom A., Monachon G., 1976. Genetics and Generalities on geese and ducks. Station expérimcntale de l'Oie, Artiguères. Document à diffusion restreinte.

Stasko J., Majna R., 1976. Comparison of correlations between body weight and liver weight in force fcd geese of four types. Zivoucisna Vyroba, 21, 853-859. 